\title{
Die manisch-depressiven Preisschwankungen auf den Finanzmärkten - Wie macht das die "unsichtbare Hand"?
}

Aktienkurse, Rohstoffpreise und Wechselkurse schwanken in einer Abfolge mehrjähriger „bull markets“ und „bear markets“ um den Bereich ihrer „Fundamentalgleichgewichte“. Gleichzeitig wird der Zeithorizont von Transaktionsentscheidungen auf diesen Märkten immer kürzer. Der Artikel zeigt, durch welche Verhaltensweisen sich kurzfristige Kursschübe zu mehrjährigen Trends akkumulieren.

\section{Problemstellung}

Zwei Entwicklungen stehen im Zentrum dieses Artikels:

- Aktien- und Wechselkurse, aber auch die auf Terminmärkten gebildeten Rohstoffpreise (insbesondere für Erdöl), entwickeln sich in einer Abfolge von mehrjährigen Trends nach oben und unten („,bull/bear markets"). Diese Preise konvergieren somit nicht gegen ihr „Fundamentalgleichgewicht", sondern „überschießen“ dieses nach oben und unten.

- Das Transaktionsvolumen auf Finanzmärkten ist in den Industrieländern auf mehr als das Hundertfache des nominalen Bruttoinlandsprodukts (BIP) angewachsen. Hauptursache dafür ist die permanente Beschleunigung des Handelns: Der Zeithorizont der meisten Transaktionsentscheidungen liegt zwischen einigen Minuten und wenigen Stunden.

Setzt man beide Entwicklungen zueinander in Beziehung, so ergibt sich ein veritables Rätsel: Wie können kurzfristige Transaktionen Kursbewegungen auslösen, die sich zu mehrjährigen „bull markets“ bzw. „bear markets“ akkumulieren? Konkreter gefragt: Welche Eigenschaften von ,asset prices" sind dafür bestimmend, dass diese Preise in mehrjährigen irregulären Zyklen um ihr realwirtschaftliches Gleichgewicht als „Gravitationszentrum“ schwanken?

Im Folgenden zeichne ich zunächst ein (hypothetisches) Bild von Transaktionsverhalten und Preisdynamik auf spekulativen Märkten, in das Antworten auf die obigen Fragen eingebettet sind. Dieses Bild habe ich (mir) in den letzten 25 Jahren auf in-

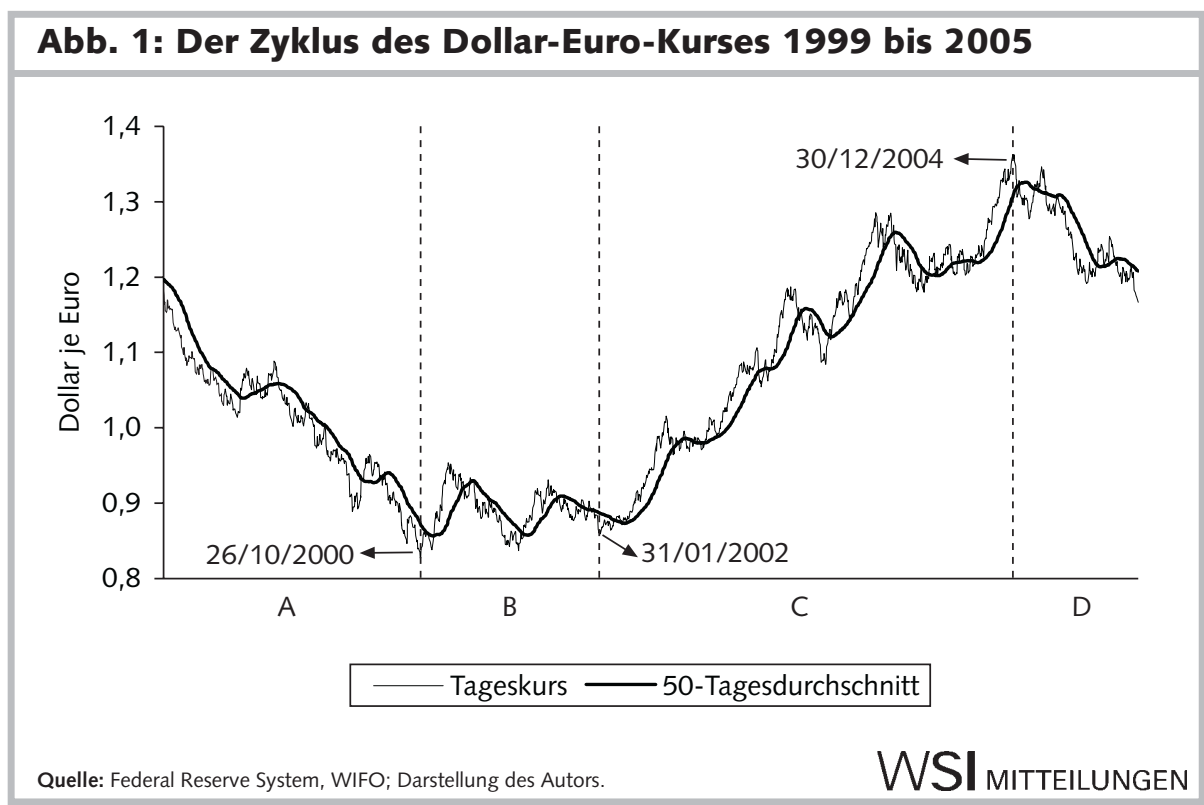

duktivem Weg erarbeitet, seine Informationsbasis umfasst u. a. Interviews mit professionellen Händlern, Tests der von ihnen verwendeten "trading systems“ sowie Analysen der Auswirkungen der „trading signals" auf die Kursentwicklung.

Im Hauptteil des Artikels werde ich für einzelne Puzzlesteine des Gesamtbilds die empirische Evidenz skizzieren, konkrete Erklärungen formulieren und diese mit den Annahmen des ökonomischen Mainstream vergleichen.

\section{Transaktionsverhalten und Preisdynamik auf Finanz- märkten im Überblick}

Kurzfristig schwanken „asset prices“ stärker als nach dem „Random-Walk-Modell“ $\mathrm{zu}$ erwarten wäre. Allerdings schwanken die Kurse fast immer um „underlying trends" (Seitwärtsbewegungen sind untypisch). Glättet man die Kurse mit Hilfe gleitender Durchschnitte, so werden die "underlying trends" offenkundig (Abbildung 1).

Das Phänomen des „trending “ wiederholt sich auf unterschiedlichen Zeitskalen. Dabei gilt: Die kurzfristige Volatilität um den Trend ist umso höher, je höher die Datenfrequenz ist (sie ist etwa bei Minutenkursen viel größer als bei Tageskursen).

Längerfristige Trends nach oben bzw. unten („bulls" und „bears") ergeben sich aus der Akkumulation von Kursschüben

Stephan Schulmeister, Dr. jur., Mag. rer. soc. oec., Mitarbeiter am Österreichischen Institut für Wirtschaftsforschung (WIFO). Arbeitsschwerpunkte: Preisdynamik auf Finanzmärkten und ihre rea/wirtschaftlichen Folgen, Zinssatz, Wachstumsrate und Staatsverschuldung. e-mail: stephan.schulmeister@wifo.ac.at 
(„runs“) auf Basis von Tagesdaten, welche über mehrere Jahre in eine Richtung länger dauern als in die Gegenrichtung (vgl. den Zyklus des Dollar/Euro-Kurses zwischen 1999 und 2005 in Abbildung 1).

Genauso resultiert ein kurzfristiger Aufwärtstrend auf Basis von Tagesdaten aus der Akkumulation von Kursschüben auf Basis von 30-Minuten-Daten, welche nach oben länger dauern als nach unten.

Auslöser von Kursschüben sind neue Informationen („news“), insbesondere über die für den jeweiligen „asset price“ relevanten Fundamentalvariablen. Treffen solche „news“ ein, schätzen die einzelnen Händler innerhalb von Sekunden ab, wie die Mehrheit der anderen Händler reagieren wird. Erwartet ein Händler, dass die Mehrheit einen Kursanstieg erwartet, so wird er das entsprechende ,asset“ kaufen (Keynes' „beauty contest“ - Keynes 1936, S. 156).

Um die Komplexität extrem rascher Spekulationsentscheidungen auf jenes Minimum zu reduzieren, das ausreicht, um Gewinne zu machen, bilden Händler auf Grund von „news“ lediglich Erwartungen über die Bewegungsrichtung von Kursen, nicht aber über ihr Gleichgewichtsniveau. So wird ein Händler bei einer überraschenden Dollarzinssenkung blitzartig auf eine Euroaufwertung setzen („Richtungserwartung“), statt das neue „Fundamentalgleichgewicht" des Euro-Dollar-Kurses zu quantifizieren.

Auf einen von „news“ ausgelösten Kursanstieg(-rückgang) folgt eine Kaskade von Kauf(Verkaufs)signalen von „trendfollowing technical trading systems“. Zuerst produzieren die besonders kursreagiblen („schnellen“) Modelle auf Basis hochfrequenter Kursdaten Handelssignale, zum Schluss die „langsamen“ Modelle auf Basis von Stunden- oder Tagesdaten. Die Umsetzung dieser „trading signals“ verstärkt in einem Rückkoppelungsprozess die Kursbewegung, sie entwickelt sich zu einem Trend.

Häufig setzt sich ein Trend fort, nachdem bereits alle technischen Modelle eine dem Trend entsprechende offene (=spekulative) Position bezogen haben. Dieses Phänomen ist in erster Linie auf den „Mitläufereffekt" von Amateuren zurückzuführen diese springen in der Regel später auf einen Trend auf als die professionellen Händler.

Je länger ein Trend anhält, desto mehr verliert er an Dynamik. Dieses „,fading-out“ nutzen die „contrarian strategies“ der technischen Analyse. Sie produzieren Verkaufs(Kauf)signale in der Spätphase eines
Aufwärts(Abwärts)trends, spekulieren also auf sein „Kippen“. Die Umsetzung dieser Signale trägt dann zu einem Richtungswechsel bei.

Die Abfolge solcher Kursschübe lässt sich auf jeder Zeitskala beobachten. Aus ihrem Zusammenwirken ergeben sich mehrjährige Trends: Minutentrends in die gleiche Richtung, unterbrochen von kürzeren Gegenbewegungen, addieren sich zu einem Stundentrend, mehrere Stundentrends zu Tagestrends usw. Auf Basis von Jahresdaten nimmt dieser Prozess die Gestalt mehrjähriger „bull markets“ und „bear markets“ an (Abbildungen 2 bis 4).

Die Tatsache, dass Kursschübe in eine Richtung langfristiger sind als in die Gegenrichtung, spiegelt die Tatsache wider, dass auf Finanzmärkten zumeist ein mittelbis längerfristiger „expectational bias“ herrscht, im professionellen Jargon als „bullishness“ bzw. „bearishness“ bezeichnet. Dieser „bias“ bedeutet, dass Marktteilnehmer „ceteris paribus“ eher eine Aufwertung bzw. eine Abwertung des jeweiligen "asset" erwarten.

Die Etablierung einer solchen asymmetrischen Marktstimmung wird von vielen Faktoren beeinflusst. So nimmt die Wahrscheinlichkeit für einen Stimmungswechsel mit dem Ausmaß des „Overshooting“ zu. Dabei wird nicht nur die Logik von Zyklen wirksam („what comes up, must come down"), sondern auch ökonomische Auswirkungen einer anhaltenden Über- oder Unterbewertung des jeweiligen ,asset“ kommen zum Tragen. So wird eine ausgeprägte Überbewertung einer Währung die Leistungsbilanz des entsprechenden Landes verschlechtern und sein Wirtschaftswachstum dämpfen. Das Umgekehrte gilt für das Land mit der unterbewerteten Währung. Je mehr sich dieses realwirtschaftliche Ungleichgewicht ausweitet, umso eher wird eine korrigierende Wechselkursentwicklung erwartet.

Auch emotionale Faktoren spielen eine wesentliche Rolle. „Geld macht sinnlich, wie uns die Erfahrung lehrt" heißt es bei Bertolt Brecht - die Erregungszustände auf Börsen bieten reichlich Anschauungsmaterial. Entscheidend dabei ist, dass die individuellen Stimmungsschwankungen auf vielfältige Weise sozial gebündelt werden (durch den „common (non)sense" der Analysten, die Ratingagenturen, die Finanznachrichtendienste, etc.).

Die Dominanz einer asymmetrischen Marktstimmung verursacht bei den Markt- teilnehmern eine selektive Wahrnehmung: Auf „news“, die der Marktstimmung entsprechen, wird viel stärker reagiert als auf gegenteilige Nachrichten.

Der durch die jeweilige Marktstimmung verursachte „bias“ in der Wahrnehmung von "news" und in dem dadurch ausgelösten Transaktionsverhalten hat folgende Konsequenzen: Kursschübe, die der Marktstimmung entsprechen, dauern länger als die Gegenbewegungen. Diese Differenz lässt ,asset prices“ über mehrere Jahre steigen oder fallen, die Abfolge der entsprechenden „bull markets“ und „bear markets“ ergibt das typische Muster der langfristigen Dynamik spekulativer Preise: Sie schwanken in irregulären Zyklen um den Bereich des realwirtschaftlichen Gleichgewichts ohne eine Tendenz, zu diesem Gleichgewicht zu konvergieren.

\section{Die langfristigen Zyklen spekulativer Preise}

Im Folgenden möchte ich die langfristigen "asset price dynamics" am Beispiel des Wechselkurses zwischen Dollar und Euro (ECU), der Aktienkurse in den USA und Deutschland sowie am Beispiel des Erdölpreises skizzieren.

Abbildung 2 verdeutlicht, wie stark der Dollar/Euro(ECU)-Wechselkurs seinen (theoretischen) Gleichgewichtswert der Kaufkraftparität international gehandelter Sachgüter und Dienstleistungen nach oben und unten „überschießt“ (zu ihrer Berechnung vgl. Schulmeister 2005a). Zwischen 1980 und 1985 wertete der Dollar drastisch auf, die Unterbewertung des ECU wurde immer größer. Darauf folgte eine neuerlich überschießende Dollarabwertung, sodass der ECU in den 1990er Jahren permanent überbewertet war. Dies wurde durch die Abwertung des ECU/Euro zwischen 1995 und 2000 korrigiert. Die mit der Rezession 2001 einsetzende Niedrigzinspolitik in den USA und ein begleitendes "talking the dollar down“ trugen wesentlich zur neuerlich überschießenden Euro-Aufwertung bei.

In den 1960er und 1970er Jahren konzentrierte sich das unternehmerische Gewinnstreben auf realwirtschaftliche Aktivitäten, gleichzeitig stagnierten die Aktienkurse. Dementsprechend ging der Börsenwert (Marktkapitalisierung) der Ak- 
Abb. 2: Wechselkurs und Kaufkraftparität von Dollar und ECU bzw. Euro, 1970-2006

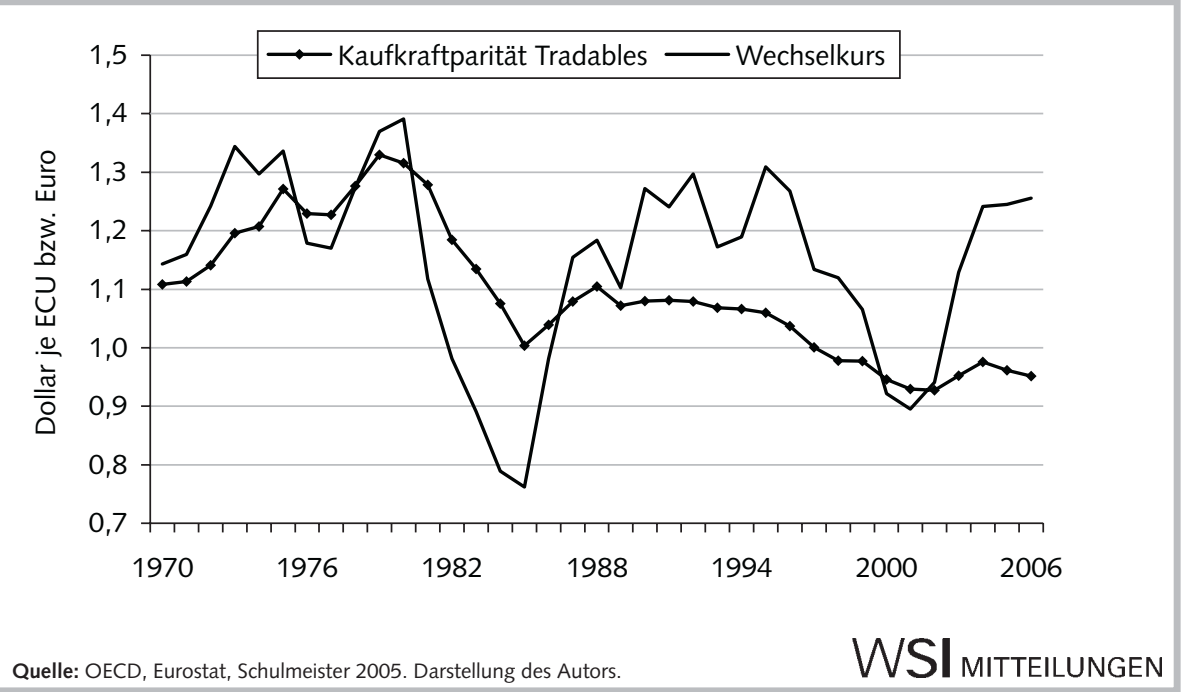

Abb. 3: Börsenwert und Netto-Gesamtwert der nicht-finanziellen Aktiengesellschaften in den USA und Deutschland, 1960-2006 - in \% -

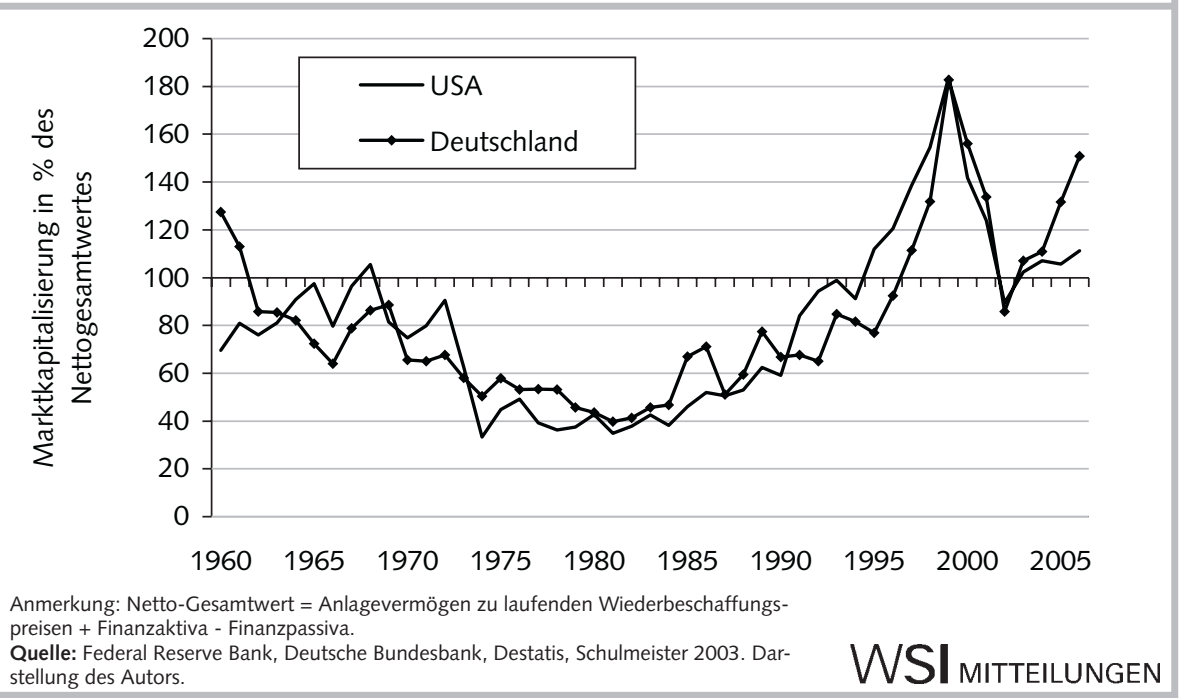

Abb. 4: Ölpreisschwankungen und Dollarkurs, 1966-2006 (1986=100)

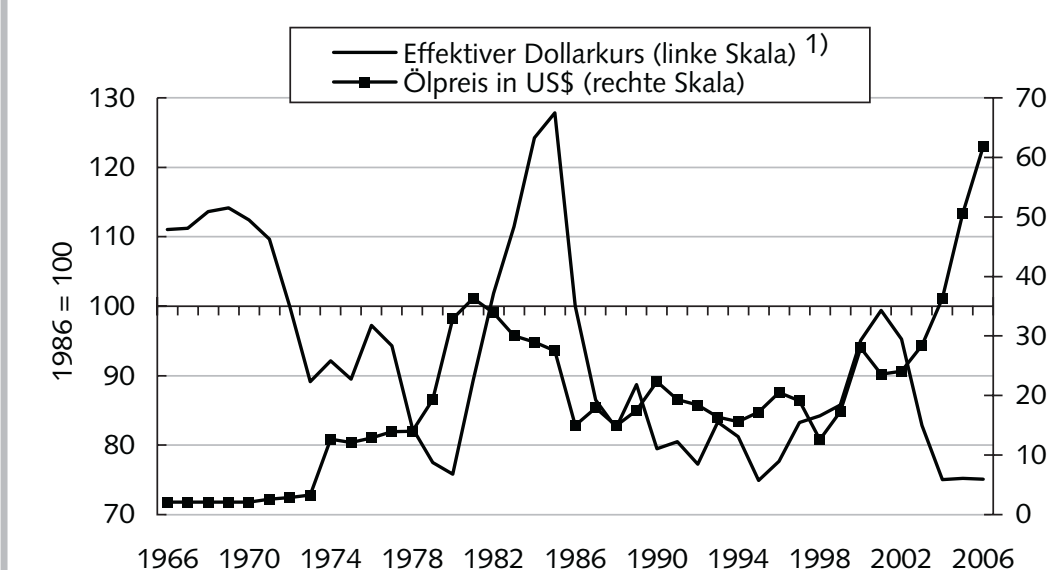

tiengesellschaften relativ zu ihrem NettoGesamtwert stark zurück (Abbildung 3). ${ }^{1}$ Ende 1980 erreichte die Unterbewertung der Aktien ihr Maximum: Der Börsenwert der Unternehmen lag in den USA und Deutschland um etwa $60 \%$ unter ihrem Netto-Gesamtwert. Der nachfolgende Aktienboom verursachte eine zunehmende Überbewertung der Kurse: Ende 1999 lag der Börsenwert der Aktiengesellschaften in den USA und Deutschland um mehr als $80 \%$ über ihrem Netto-Gesamtwert ( $A b$ bildung 3). Diese Diskrepanz trug wesentlich zum "Kippen“ des "bull market" in einen „bear market“ bei: Ende 2002 waren die Aktienkurse wieder (leicht) unterbewertet. Ab dem Frühjahr 2003 begannen die Kurse neuerlich stark zu steigen. Da gleichzeitig die Realkapitalbildung in Deutschland stagnierte, in den USA aber expandierte, ließ der „bull market“ die Diskrepanz zwischen Börsenwert und NettoGesamtwert der Unternehmen in Deutschland viel stärker steigen als in den USA ( $A b$ bildung 3). Im Sommer 2007 dürfte erneut ein „bear market“ begonnen haben.

Da Erdöl eine erschöpfbare Ressource ist, deren Preis auch von nicht-ökonomischen Faktoren stark beeinflusst wird, lässt sich sein „fundamentaler" Gleichgewichtspreis nicht genau abschätzen. Allerdings ist es ausgeschlossen, dass dieser so stark schwankt wie der tatsächliche Erdölpreis (Abbildung 4). Vielmehr lässt die Interaktion von „,news-based trading“ und „technical trading" auf den Ölterminmärkten auch diesen ,asset price“ nach oben und unten „überschießen“. Die längerfristigen Ölpreistrends werden überdies auch von den Schwankungen des Dollarkurses mitbestimmt: Da der US-Dollar die globale Leitwährung darstellt, notiert Erdöl ausschließlich in Dollar. Folglich entwertet jede ausgeprägte Dollarabwertung die realen Exporterlöse der Erdölproduzenten und erhöht so für sie den Anreiz, den Preis hinaufzusetzen (Schulmeister 2000). Ist ihre Marktposition stark - wie in den 1970er Jahren sowie in jüngster Zeit - so können sie eine „überkompensierende“ Ölpreissteigerung durchsetzen (Abbildung 4).

Der Netto-Gesamtwert ergibt sich als Summe des Realkapitals zu laufenden Marktpreisen plus Finanzaktiva minus Finanzpassiva - er wird als "proxy" für den "fundamentalen Unternehmenswert ${ }^{\prime}$ verwendet (zu seiner Berechnung vgl. Schulmeister 2003) 
Die Gleichgewichtsökonomie unter rationalen Erwartungen kann zyklische Schwankungen von ,asset prices“ um ihr fundamentales Gleichgewicht nicht erklären. ${ }^{2}$ Denn sie kennt nur zwei Preispfade: Konvergenz zum fundamentalen Gleichgewicht oder einen „bubble“. Somit ist genau jenes Phänomen, das empirisch am besten beobachtbar ist und von Marktteilnehmern als Abfolge von „bulls“ und „bears“ klar benannt wird, in der herrschenden Theorie nicht vorgesehen.

In den empirischen Wechselkursstudien wird das „purchasing power partity puzzle“ in erster Linie darin gesehen, dass Wechselkurse so viele Jahre brauchen, bis sie wieder das fundamentale Gleichgewicht erreichen („mean reversion“). Der vorangegangene Prozess des „overshooting “ wird hingegen auf Schocks zurückgeführt und daher nicht erklärt (Rogoff 1996; Sarno/ Taylor 2002; Taylor/Taylor 2004). Dadurch kann der Mainstream die wechselseitige Bedingtheit von „overappreciations" und "overdepreciations" nicht wahrnehmen.

Auch Studien zur Aktienkursdynamik konzentrieren sich auf einzelne Anomalien wie den „momentum effect“ (als Folge der Kurstrends) oder den „reversal effect" (als Folge des Richtungswechsels von Trends). Sie werden aber nicht in den Gesamtkontext der zyklischen Schwankungen von "asset prices" gestellt (für einen Überblick zur empirischen Literatur über die Aktienkursdynamik vgl. Campbell 2000; Cochrane 1999; Lo/MacKinlay 1999; Shiller 1999). Maßgeblich dafür ist, dass auch die „behavioral finance" die Gleichgewichtstheorie als Referenzmodell verwendet - ihr widersprechende Beobachtungen können daher nur als „Anomalien“ wahrgenommen werden.

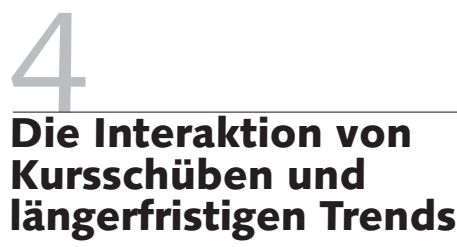

Zwei markante Trends prägten den Zyklus des Dollar/Euro-Kurses 1999/2005: Der Abwertungstrend zwischen Januar 1999 und Oktober 2000 und der noch längere Aufwertungstrend zwischen Januar 2002 und Dezember 2004 (Perioden A und C in Abbildung 1). Der Abwertungstrend wurde in drei, der Aufwertungstrend in vier Kursschüben („underlying trends“) reali-

\begin{tabular}{|c|c|c|c|c|c|c|}
\hline Periode & $\begin{array}{l}\text { Dauer } \\
\text { in Tagen }\end{array}$ & $\begin{array}{l}\text { Veränderung } \\
\text { im Kursniveau } \\
\text { in Cents }\end{array}$ & $\begin{array}{l}\text { Länge des } \\
\text { Kurspfades } \\
\text { in Cents }{ }^{1)}\end{array}$ & $\begin{array}{c}\text { Kurs- } \\
\text { veränderung } \\
\text { pro Tag } \\
\text { in Cents } \\
\text { (Anstieg) }\end{array}$ & $\begin{array}{l}\text { Länge des } \\
\text { Kurspfades } \\
\text { pro Tag } \\
\text { in Cents }\end{array}$ & $\begin{array}{c}\text { Veränderung } \\
\text { im Kursniveau } \\
\text { relativ zur } \\
\text { Länge des } \\
\text { Kurspfades }\end{array}$ \\
\hline \multicolumn{7}{|c|}{ Originaldaten } \\
\hline A & 471 & $-34,4$ & 226,0 & $-0,07$ & 0,480 & $-0,15$ \\
\hline B & 324 & 2,8 & 156,4 & 0,01 & 0,483 & 0,02 \\
\hline C & 755 & 50,1 & 405,3 & 0,07 & 0,537 & 0,12 \\
\hline $\mathrm{D}$ & 224 & $-18,7$ & 123,0 & $-0,08$ & 0,549 & $-0,15$ \\
\hline \multicolumn{7}{|c|}{ Gleitender Fünf-Tagesdurchschnitt } \\
\hline$A$ & 467 & $-33,5$ & 110,0 & $-0,07$ & 0,236 & $-0,30$ \\
\hline B & 320 & 2,1 & 70,2 & 0,01 & 0,219 & 0,03 \\
\hline C & 751 & 49,3 & 183,0 & 0,07 & 0,244 & 0,27 \\
\hline D & 210 & $-12,5$ & 30,1 & $-0,06$ & 0,144 & $-0,41$ \\
\hline \multicolumn{7}{|c|}{$\begin{array}{l}\text { 1) Absolute tägliche Kursänderungen, kumuliert. } \\
\text { Periode A: } 1 / 1 / 1999 \text { bis } 25 / 10 / 2005 \text {, Periode B: } 26 / 10 / 2000 \text { bis } 31 / 1 / 2002 \text {, } \\
\text { Periode C: } 3 / 1 / 2002 \text { bis } 30 / 12 / 2004 \text {, Periode D: } 31 / 12 / 2004 \text { bis } 14 / 11 / 2005 \text {. }\end{array}$} \\
\hline
\end{tabular}

\begin{tabular}{|c|c|c|c|c|c|c|}
\hline \multirow[b]{2}{*}{ Periode } & \multicolumn{3}{|c|}{ Aufwertungsruns } & \multicolumn{3}{|c|}{ Abwertungsruns } \\
\hline & Anzahl & $\begin{array}{c}\text { Durch- } \\
\text { schnittliche } \\
\text { Dauer } \\
\text { in Tagen }\end{array}$ & $\begin{array}{c}\text { Durch- } \\
\text { schnittlicher } \\
\text { Anstieg 1) }\end{array}$ & Anzahl & $\begin{array}{c}\text { Durch- } \\
\text { schnittliche } \\
\text { Dauer } \\
\text { in Tagen }\end{array}$ & $\begin{array}{l}\text { Durch- } \\
\text { schnittlicher } \\
\text { Anstieg1) }\end{array}$ \\
\hline & \multicolumn{6}{|c|}{ Originaldaten } \\
\hline A & 113 & 1,8 & 0,474 & 113 & 2,4 & $-0,484$ \\
\hline B & 79 & 2,0 & 0,510 & 79 & 2,1 & $-0,457$ \\
\hline C & 210 & 1,9 & 0,557 & 209 & 1,7 & $-0,513$ \\
\hline \multirow[t]{2}{*}{ D } & 57 & 1,7 & 0,527 & 58 & 2,2 & $-0,567$ \\
\hline & \multicolumn{6}{|c|}{ Gleitender Fünf-Tagesdurchschnitt } \\
\hline$A$ & 44 & 3,8 & 0,229 & 45 & 6,6 & $-0,240$ \\
\hline B & 37 & 4,0 & 0,246 & 36 & 4,8 & $-0,199$ \\
\hline C & 70 & 6,8 & 0,245 & 68 & 4,1 & $-0,242$ \\
\hline D & 9 & 8,4 & $-0,116$ & 10 & 13,4 & $-0,159$ \\
\hline \multicolumn{7}{|c|}{$\begin{array}{l}\text { 1) Durchschnittliche Veränderung des Kursniveaus pro Tag in Cents. } \\
\text { Periode A: } 1 / 1 / 1999 \text { bis } 25 / 10 / 2005 \text {, Periode B: } 26 / 10 / 2000 \text { bis } 31 / 1 / 2002 \text {, } \\
\text { Periode C: } 3 / 1 / 2002 \text { bis } 30 / 12 / 2004 \text {, Periode D: } 31 / 12 / 2004 \text { bis } 14 / 11 / 2005 \text {. }\end{array}$} \\
\hline
\end{tabular}

siert, jeweils unterbrochen durch relativ kurze Gegenbewegungen. Der gleitende 50-Tagedurchschnitt verdeutlicht dieses Entwicklungsmuster. Wie Abbildung 3 zeigt, schwanken die Tageskurse erheblich um die jeweiligen ,, underlying trends", insbesondere in der Abwertungsphase A.

Um herauszufinden, auf welche Weise sich die Veränderungen der Tageskurse zu mehrjährigen Auf- und Abwertungstrends akkumulieren, möchte ich zunächst den Wechselkurspfad vermessen (Tabelle 1). ${ }^{3}$

In Periode A verliert der Euro in 471 Handelstagen 34,4 Dollar-Cent an Wert (0,07 pro Tag). Wegen der vielen kurzfristigen Auf- und Abwärtsbewegungen ist die Länge des Gesamtpfads des Euro (= absolute Kursänderungen, kumuliert) viel größer als dieser „Höhenunterschied“, sie beträgt 226,0 Cent $(0,48$ pro Tag). Im Fall einer monotonen Abwertung wären die
Veränderung im Kursniveau und die Länge des Kurspfades gleich. Die Relation dieser beiden Größen stellt daher einen Indikator für das Ausmaß der Gegenbewegungen während eines Kurstrends dar. Diese Relation ist in den drei Trendperioden

2 Modelle zur Erklärung, warum „asset prices" systematisch um den Bereich ihres "fundamentalen“ Gleichgewichts schwanken, finden sich in Schulmeister 1987 und Frydman/Goldberg 2007. Zu den "long swings" des Dollarkurses siehe auch Engel/Hamilton 1990.

3 Die im Folgenden präsentierten "stylized facts zur Dynamik des Dollar/Euro-Kurses stammen aus einem Forschungsprojekt, das ich derzeit noch bearbeite. Seine Gesamtergebnisse werden 2008 unter dem Titel "Technical Trading and Trends in the Euro Exchange Rate" publiziert (dieses Forschungsvorhaben wird unter der Projektnummer 11989 vom Jubiläumsfonds der Österreichischen Nationalbank dankenswerterweise unterstützt). 


\begin{tabular}{|c|c|c|c|c|c|c|c|c|c|}
\hline & \multirow{3}{*}{$\begin{array}{l}\text { Dauer in } \\
\text { Tagen }\end{array}$} & \multicolumn{2}{|c|}{ Aufwertungsruns } & \multicolumn{2}{|c|}{ Abwertungsruns } & \multicolumn{2}{|c|}{ Aufwertungsruns } & \multicolumn{2}{|c|}{ Abwertungsruns } \\
\hline & & $\begin{array}{l}\text { Beob- } \\
\text { achtet }\end{array}$ & $\begin{array}{c}\text { Erwartet } \\
\text { unter RWH }\end{array}$ & $\begin{array}{l}\text { Beob- } \\
\text { achtet }\end{array}$ & $\begin{array}{c}\text { Erwartet } \\
\text { unter RWH }\end{array}$ & $\begin{array}{l}\text { Beob- } \\
\text { achtet }\end{array}$ & $\begin{array}{c}\text { Erwartet } \\
\text { unter RWH }\end{array}$ & $\begin{array}{l}\text { Beob- } \\
\text { achtet }\end{array}$ & $\begin{array}{c}\text { Erwartet } \\
\text { unter RWH }\end{array}$ \\
\hline & & \multicolumn{4}{|c|}{ Periode A: $1 / 1 / 1999$ bis $25 / 10 / 2000$} & \multicolumn{4}{|c|}{ Periode C: $1 / 2 / 2002$ bis $30 / 12 / 2004$} \\
\hline \multirow[t]{4}{*}{ Originaldaten } & $1-2$ & 93 & 88,7 & $69 * * *$ & 88,8 & $163 * *$ & 141,9 & $177 * * *$ & 141,8 \\
\hline & $3-6$ & $20 * *$ & 27,7 & $42 * * *$ & 27,5 & 43 & 44,3 & $32 * * *$ & 44,3 \\
\hline & 37 & $0 *$ & 1,8 & 2 & 1,8 & 4 & 2,9 & $0 * *$ & 2,9 \\
\hline & Alle & 113 & 118,2 & 113 & 118,2 & $210 * * *$ & 189,0 & $209 * * *$ & 189,1 \\
\hline \multirow{4}{*}{$\begin{array}{l}\text { Gleitender Fünf- } \\
\text { Tagesdurchschnitt }\end{array}$} & $1-6$ & 37 & 35,9 & $27^{*}$ & 36,0 & $44 * *$ & 57,2 & 53 & 57,1 \\
\hline & $7-14$ & $5 * *$ & 10,4 & 11 & 10,4 & 18 & 16,6 & 15 & 16,8 \\
\hline & 315 & 2 & 2,0 & $7 * * *$ & 2,0 & $8 * * *$ & 3,3 & $0 * *$ & 3,2 \\
\hline & Alle & 44 & 48,4 & 45 & 48,4 & 70 & 77,1 & $68 *$ & 77,1 \\
\hline \multirow{4}{*}{$\begin{array}{l}\text { Gleitender 20-Tages- } \\
\text { durchschnitt }\end{array}$} & $1-14$ & 16 & 18,0 & $11 *$ & 18,0 & 29 & 28,7 & 31 & 28,7 \\
\hline & $15-34$ & 3 & 4,1 & 5 & 4,1 & 4 & 6,5 & 6 & 6,6 \\
\hline & 335 & 0 * & 1,4 & $4 * * *$ & 1,4 & $5 * *$ & 2,4 & $0 * *$ & 2,3 \\
\hline & Alle & 19 & 23,5 & 20 & 23,5 & 38 & 37,5 & 37 & 37,5 \\
\hline \multicolumn{10}{|c|}{$\begin{array}{l}\text { Sowohl die beobachtete Wechselkursreihe als auch die } 1000 \text { Zufallsreihen sind mit den angeführten gleitenden Durchschnitten geglättet. Danach werden die Runs nach iher Dauer klassifiziert. Die erste } \\
\text { Differenzen der } 1000 \text { Zufallsreihen haben die gleiche Varianz wie die beobachteten Wechselkurse in der jeweiligen Periode. *(**** ***) kennzeichnen die Signifikanz der Abweichung der beobachteten } \\
\text { Anzahl von Runs von ihrem Erwartungswert unter der "Random-Walk-Hypothese" (RWH) mit Irrtumswahrscheinlichkeiten kleiner } 10 \%(5 \%, 1 \%) \text {. }\end{array}$} \\
\hline
\end{tabular}

(A, C, D) annähernd gleich (letzte Spalte in Tabelle 1).

Wiederholt man diese "Vermessung" für die mit einem gleitenden Fünf-Tagesdurchschnitt geglättete Kursreihe, so verringert sich die Länge des Kurspfades in allen vier Perioden auf weniger als die Hälfte. Dafür sind zwei Gründe maßgeblich: Erstens ist ein Großteil der Kursfluktuationen klein und von kurzer Dauer. Zweitens schwankt der Eurokurs fast immer um einen „underlying trend“ (dies gilt auch für die Periode B).

Im nächsten Schritt untersuche ich, auf welche Weise die Akkumulation der monotonen Kursbewegungen („runs“) nach oben bzw. unten mehrjährige Trends hervorbringt (Tabelle 2). In Periode A wurde die Euroabwertung in erster Linie dadurch realisiert, dass die Runs nach unten um ein Drittel länger dauerten als die Runs nach oben (2,4 gegenüber 1,8 Tage); der durchschnittliche Anstieg von Abwertungs- und Aufwertungsruns war annähernd gleich. Dieses Muster gilt auch analog für die beiden anderen Trendperioden C und D.

Besonders markant tritt dieses Muster auf Basis der durch einen gleitenden FünfTagesdurchschnitt geglätteten Kursreihe zu Tage: Mehrjährige Aufwertungen (Abwertungen) werden dadurch realisiert, dass Kursschübe (Runs) nach oben (unten) länger dauern als die Gegenruns, und nicht dadurch, dass Aufwertungs(Abwertungs)runs steiler sind als die Gegenbewegungen. Dieses Ergebnis hatte sich bereits bei „Vermessung "des Pfades des DM/\$-Kurses zwischen 1980 und 1986 gezeigt (Schulmeister 1987).
Nun wird der Zusammenhang zwischen der Verteilung der Runs nach ihrer Dauer und dem langfristigen Abwertungsbzw. Aufwertungstrend des Dollar/EuroKurses in Periode A bzw. C untersucht. Wie Tabelle 3 zeigt, wurde die Euro-Abwertung (Periode A) dadurch realisiert, dass in dieser Phase kurze Runs (mit einer Dauer bis zu zwei Tagen) häufiger aufwärts als abwärts gerichtet waren (93 gegenüber 69), während mittlere Runs (drei bis sechs Tage) und längere Runs (länger als sechs Tage) häufiger nach unten als nach oben gingen. Für die Aufwertungsphase (Periode C) ergibt sich analog: Längere Runs sind häufiger Aufwertungs- als Abwertungsruns, kurze Runs sind häufiger Abwertungsruns.

Um die Robustheit der bisherigen Ergebnisse zu testen, habe ich für die beiden Trendphasen je 1.000 Zufallsreihen generiert, deren erste Differenzen die gleiche Varianz aufweisen wie die beobachtete Wechselkursreihe („random walks without drift"). Dadurch soll geprüft werden, in welchen Längenklassen von Runs und auf Basis welcher gleitender Durchschnitte die Anzahl der beobachteten Kursschübe signifikant von ihren Erwartungswerten unter der „Random-Walk-Hypothese“ (RWH) abweichen.

Auf Basis der Originaldaten traten in der Aufwertungsperiode C signifikant mehr kurze Runs als bei den Zufallsreihen auf; dies gilt insbesondere für Abwertungsruns. Gleichzeitig ergaben sich signifikant weniger Abwertungsruns mittlerer Dauer ( $\mathrm{Ta}$ belle 3). In der Abwertungsphase A traten umgekehrt signifikant weniger kurze, aber signifikant mehr mittlere Abwertungsruns auf. Gleichzeitig war die Zahl der mittleren Aufwertungsruns signifikant kleiner als bei einem Zufallsprozess.

Auf Basis geglätteter Reihen verlagert sich die Abweichung der Anzahl von Runs der beobachteten Reihe von den Erwartungswerten unter der RWH zu den persistenten Kursschüben (Runs mit einer Dauer von mehr als 14 bzw. mehr als $34 \mathrm{Ta}$ gen im Fall eines gleitenden 5- bzw. 20Tagedurchschnitts): In der Abwertungsperiode $\mathrm{A}$ traten hochsignifikant mehr lange Abwertungsruns auf, in der Aufwertungsperiode traten analog (hoch)signifikant mehr lange Aufwertungsruns auf (Tabelle 3). Gleichzeitig ergaben sich in der Aufwertungsphase C signifikant weniger persistente Kursschübe nach unten.

Somit ergibt sich ein klares Muster, auf welche Weise mehrjährige Wechselkurstrends realisiert werden: Kurzfristig schwanken die Kurse um „underlying trends“; diese persistenten Kursschübe dauern in eine Richtung länger als in die Gegenrichtung. Als Folge der hohen kurzfristigen Volatilität tritt dieses Muster nur auf Basis geglätteter Kursreihen markant zu Tage. ${ }^{4}$

Dieses Muster in der Dynamik spekulativer Preise widerspricht den Erwartungen der (noch) herrschenden „efficient markets hypothesis“. Denn nach dieser

4 Erste Berechnungen zeigen, dass sich längerfristige Trends der Aktienkurse (S\&P 500, DAX) sowie des Erdölpreises (Brent Futures) nach dem gleichen Muster entwickeln. 
Theorie reflektiert jeder „asset price“ alle zum jeweiligen Zeitpunkt vorhandenen Informationen, Preisveränderungen ergeben sich aus neuen Informationen und folgen daher einem Zufallsprozess (zur neueren Debatte über die „efficient market hypothesis" vgl. LeRoy 1989; Shiller 2003; Lo 2004).

\section{Die kurzfristige Kursdynamik}

Eine Analyse des „trending“ von Wechselkursen auf Basis von Daten im 30-Minutenintervall brachte folgende Ergebnisse:

- Auf Basis der Originalkurse ergaben sich signifikant mehr kurze Runs als nach der RWH erwartet, hingegen signifikant weniger längere Runs. Auch insgesamt ist die Zahl der Runs signifikant höher als bei einem Zufallsprozess. Dementsprechend ist die durchschnittliche Dauer der beobachteten Runs kürzer, als nach der RWH zu erwarten wäre.

- Auf Basis der mit gleitenden Durchschnitten geglätteten Reihen zeigt sich ein ganz anderes Bild. Es ergaben sich weniger kurze Runs als unter der RWH zu erwarten war, hingegen signifikant mehr längere Runs.

Fazit: Auf Basis von Hochfrequenzdaten ist die Volatilität der kurzen Runs des Dollar/ Euro-Wechselkurses (noch) höher als im Fall von Zufallsreihen. Allerdings folgen (auch) diese kurzen „ups and downs“ einem „underlying trend“. Deshalb ergeben sich auf Basis geglätteter Kursreihen weniger kurze und mehr lange Runs. Letztere dauern während einer $\mathrm{Ab}$ (Auf)wertungsphase nach unten (oben) länger als die Gegenbewegungen. Aus der Akkumulation unterschiedlich langer Kursschübe resultiert ein längerfristiger $\mathrm{Ab}$ - oder Aufwertungsprozess.

\section{Die Rolle technischer Spekulationssysteme}

Der Begriff „technische Analyse“ umschreibt all jene Handelstechniken, die versuchen, das sich auf den unterschied- lichen Zeitskalen wiederholende Phänomen des "trending"von ,asset prices" profitabel auszunutzen. Dabei sind zwei Ansätze zu unterscheiden: „Trend-following systems" versuchen, in einer Frühphase eines Trends ,aufzuspringen“, „contrarian systems" spekulieren umgekehrt in der Spätphase eines Trends auf einen Richtungswechsel (für eine umfassende Darstellung vgl. Kaufman 1987; eine knappe Darstellung der wichtigsten quantitativen „trading rules“ findet sich in Schulmeister 2007b).

In den vergangenen 20 Jahren wurde die „technische Analyse“ verwendeten Handelstechnik (für den Devisenmarkt vgl. Cheung et al. 2004; Gehrig/ Menkhoff 2006; Menkhoff/Taylor 2007). Dazu haben mehrere Faktoren beigetragen: Die „universelle“ Einsetzbarkeit dieser Systeme (auf jeder Zeitskala), die Beschleunigung beim Zugang zu „real time data“ und bei der Umsetzung von "trading signals“ (insbesondere durch das Internet) sowie die stetige Verbesserung von computergestützter „trading software“.

Abbildung 1 zeigt, wie ein besonders einfaches „moving-average-model“ (MAModell) Wechselkurstrends ausnutzen kann: Wenn immer der aktuelle Kurs den gleitenden 50-Tages-Durchschnitt von oben (unten) durchbricht, wird verkauft (gekauft). Allerdings: Die essentielle „nonrandomness“ von „asset prices“, nämlich das häufige Auftreten von Trends auf unterschiedlichen Zeitskalen, ist nicht stabil. Daraus folgt etwa im Hinblick auf MAModelle, dass die Länge der gleitenden Durchschnitte, welche die „underlying trends" optimal ausnutzen, sich in der Zeit verändert und ex ante nicht herausgefunden werden kann.

Dies bedeutet zweierlei: Einerseits bietet das ,trending “von ,asset prices“"Chance und Anreiz, dieses durch technische Spekulationsmodelle profitabel auszubeuten, andererseits zeigt sich, dass die hohen Profite, die man aufgrund von ex-post-Simulationen technischer Modelle erwartet, ex ante nicht zu erzielen sind.

Eine Eigenschaft solcher Modelle erweist sich als universell gültig, also für alle „asset prices“, Zeitskalen und Modelltypen: Die Dauer der profitablen Positionen ist immer markant länger als die Dauer der Verlustpositionen. Diese Eigenschaft reflektiert das "trending“ von "asset prices“ als ihre fundamentale "non-randomness“ (Schulmeister 2005b; 2006; 2007a; 2007b).
Zwischen dem „trending“ spekulativer Preise und der Verwendung technischer Handelssysteme besteht eine Wechselwirkung: Jeder einzelne Trader versucht, mit seinem ,individuellen“ Modell (spezifiziert durch die Modellparameter und die präferierte Zeitskala) Preistrends profitabel auszubeuten, umgekehrt verstärkt und verlängert die Sequenz technischer Kaufbzw. Verkaufssignale der unterschiedlichen „trend-following systems“ vieler einzelner Händler jeden Aufwertungs- bzw. Abwertungstrend in seiner Frühphase. In der Spätphase tragen wiederum die „contrarian systems“ zu einem „Kippen“ des Trends bei (Schulmeister 2006; 2007b).

\section{Schlussbemerkung}

Die zentrale Aussage lautet: Spekulative Preise entwickeln sich in „Schüben“, welche über einen längeren Zeitraum in eine Richtung länger dauern als die Gegenbewegungen. Die Akkumulation dieser Kursschübe resultiert in mehrjährigen Aufwertungsoder Abwertungstrends. Die Sequenz solcher „bull markets“ und „bear markets“ bildet langfristige, irreguläre Zyklen, in denen "asset prices" um ihr Fundamentalgleichgewicht als „Gravitationszentrum“ schwanken.

Die entsprechenden Beobachtungen sind geradezu mit „freiem Auge" erkennbar (Abbildungen 1 bis 4). Außerdem ist offensichtlich, dass die Akteure zumeist nur „Richtungserwartungen“ bilden („bullish/ bearish“), um das „trending“ von Kursen auszunutzen.

Gleichzeitig dämpfen die „manischdepressiven" Schwankungen so bedeutender Preise wie Wechselkurse, Rohstoffpreise und Aktienkurse die realwirtschaftliche Entwicklung auf doppelte Weise: Erstens sind Turbulenzen auf den Finanzmärkten wichtige Ursachen für Rezessionen (wie etwa 2000/2004, als der Verfall der Aktienkurse und der Anstieg von Erdölpreis und Eurokurs wesentlich zum Wirtschaftsabschwung in Europa beitrug). Zweitens verlagert sich das (unternehmerische) Gewinnstreben im „business as usual“ von realwirtschaftlichen Aktivitäten zu Finanzveranlagung und -spekulation, was wesentlich zur Wachstumsabschwächung seit Anfang der 1970er Jahre beigetragen hat (Schulmeister 2007c). 
Trotz dieser Beobachtungen und (plausiblen) Vermutungen gilt: Weder im wirtschaftswissenschaftlichen Mainstream (einschließlich der „behavioral finance“) noch in der (post-)keynesianischen Tradition wird folgende Hypothese untersucht: "Asset prices" schwanken mit großer Amplitude um den Bereich des Fundamentalgleichgewichts ohne jede Tendenz, dorthin zu konvergieren.

Dass sich der ökonomische Mainstream nicht konkret mit dieser Hypothese auseinandersetzt, ist wegen der damit verbundenen „kognitiven Dissonanz" verständlich. Wenn nämlich die „freiesten“ aller Märkte, die Finanzmärkte, systematisch falsche Preissignale setzen, dann kollabiert das gesamte Theoriegebäude. Dieses aber hat man in mühevollster Restaurationsarbeit in den letzten drei Jahrzehnten wieder aufgerichtet.

Genau dies sollte aber für „Keynesianer" ein Anreiz sein, sich verstärkt mit der Preisbildung auf ,asset markets“ und ihren makroökonomischen Konsequenzen auseinanderzusetzen. Keynes selbst hat dazu wichtige Anregungen für weitere Forschungen gegeben. Sie wurden aber in den 1950er und 1960er Jahren nicht aufgegriffen, wohl deshalb, weil die Finanzmärkte damals „ruhig gestellt" wurden. In den 1970er Jahren und danach war dieses „Erbe“ von Keynes schon in Vergessenheit geraten. Die Zeiten sind günstig, es wieder zu entdecken.

\section{LITERATUR}

Campbell, J. Y. (2000): Asset pricing at the millenium, in: Journal of Finance 4, S. 1515-1567

Cheung, Y. W./Chinn, M. D./Marsh, I. W. (2004): How do UK-Based foreign exchange dealers think their market operates?, in: International Journal of Finance and Economics 4, S. 289-306

Cochrane, J. H. (1999): New Facts in Finance, NBER Working Paper 7169, June

Engel, C./Hamilton, J. D. (1990): Long swings in the dollar: are they in the data and do markets know it?, in: American Economic Review 4, S. $689-713$

Frydman, R./Goldberg, M. D. (2007): Imperfect Knowledge Economics: Exchange Rates and Risk, Princeton University Press, Princeton, New Jersey

Gehrig, T./Menkhoff, L. (2006): Extended evidence on the use of technical analysis in foreign exchange, in: International Journal of Finance and Economics 4, S. 327-338

Kaufman, P. J. (1987): The New Commodity Trading Systems and Methods, New York

Keynes, J. M. (1936): The General Theory of Employment, Income and Money, London

LeRoy, S. F. (1989): Efficient capital markets and martingales, in: Journal of Economic Literature 4, S. 1583-1621

Lo, A. W. (2004): The adaptive market hypothesis, in: The Journal of Portfolio Management, 30th Anniversary Issue, S. 15-29

Lo, A. W./MacKinlay, A. C. (1999): A Non-Random Walk Down Wall Street, Princeton University Press, Princeton, New Jersey

Menkhoff, L./Taylor, M. P. (2007): The obstinate passion of foreign exchange professionals: technical analysis, forthcoming in Journal of Economic Literature

Rogoff, K. (1996): The purchasing power parity Puzzle, in: Journal of Economic Literature 2, S. 647-668.

Sarno, L.,/Taylor, M. P. (2002): Purchasing power parity and the real exchange rate, IMF Staff Papers 1, S. 65-105
Schulmeister, S. (1987): An Essay on Exchange Rate Dynamics, Wissenschaftszentrum Berlin. Download: http://stephan.schulmeister.ac.at/ Schulmeister, S. (2000): Globalization without global money: the double role of the dollar as national currency and as world currency, in: Journal of Post Keynesian Economics 3, S. 365-395

Schulmeister, S. (2003): Aktienkursdynamik und Realkapitalbildung in den USA und Deutschland, WIFO-Studie, Wien. Download: http://stephan. schulmeister.ac.at/

Schulmeister, S. (2005A): Purchasing Power Parities, Exchange Rates and International Price Competitiveness, WIFO-Studie, Wien. Download: http://stephan.schulmeister.ac.at/

Schulmeister, S. (2005B): Components of the Profitability of Technical Currency Trading, WIFO Working Paper 263, Download: http://stephan.schulmeister.ac.at/; forthcoming in Applied Financial Economics Schulmeister, S. (2006): The interaction between technical currency trading and exchange rate fluctuations, in: Finance Research Letters 2 , S. $212-233$

Schulmeister, S. (2007A): The Profitability of Technical Stock Trading has Moved from Daily to Intraday Data, WIFO Working Paper 289, Download: http://stephan.schulmeister.ac.at/

Schulmeister, S. (2007B): The Interaction between the Aggregate Behavior of Technical Trading Systems and Stock Price Dynamics, WIFO Working Paper 290, Download: http://stephan.schulmeister.ac.at/ Schulmeister, S. (2007C): Finanzspekulation, Arbeitslosigkeit und Staatsverschuldung, in: Intervention 1, S. 73-97

Shiller, R. J. (1999): Human Behavior and the Efficiency of the Financial System, in: Taylor, J./Woodford, W.: Handbook of Macroeconomics I, Amsterdam, North-Holland

Shiller, R. J. (2003): From efficient markets theory to behavioral finance, in: Journal of Economic Perspectives 1, S. 83-104

Taylor, A. M./Taylor, M. P. (2004): The purchasing power parity debate, in: Journal of Economic Perspectives 4, S. 135-158 